\title{
Datos preliminares sobre la abundancia relativa y caracterización del hábitat de Tayassu pecari (Artiodactyla: Tayassuidae) en los municipios del Medio Baudó y Cértegui en el Departamento del Chocó, Colombia
}

\section{Preliminary data on relative abundance and habitat characterization of Tayassu pecari (Artiodactyla: Tayassuidae) in the municipalities of Medio Baudo and Certegui in the department of the Choco, Colombia}

\author{
Leison Palacios-Mosquera ${ }^{1 *}$, Cateryne Parra lbarguen ${ }^{2}$, Hugo Mantilla-Meluk ${ }^{1,3}$
}

\section{RESUMEN}

Con el fin de estimar la abundancia relativa de Tayassu pecari en el Chocó Central, un total de $16.4 \mathrm{~km}$ de transecto lineal fueron muestreados en los municipios Medio Baudó y Cértegui. Se documentaron 42 registros de presencia, resultando en una abundancia relativa de 3.0 individuos $/ \mathrm{km}$, con intervalos de confianza de 2.7 a 4.0 individuos $/ \mathrm{km}$. El área de estudio se caracterizó por su heterogeneidad en cobertura vegetal. Reportamos 42 especies arbóreas asociadas a los transectos analizados, de las cuales el 48\% corresponden a especies en géneros previamente reportados en la dieta de $\mathrm{T}$. pecari. Se destacan en número las palmas que suman el $20 \%$ de las especies registradas, con Socratea sp., sumando el $8.57 \%$ de los elementos arborescentes. La minería tecnificada y la expansión de la frontera agrícola de cultivos no tradicionales son identificados como los principales factores que amenazan la supervivencia y conservación del tatabro en la zona de estudio.

Palabras clave: Abundancia; Caracterización ecológica; Chocó; Conservación; Tatabro sin collar; Transectos.

\section{ABSTRACT}

To evaluate the relative abundance of Tayassu pecari at the Central Choco region, $16.4 \mathrm{~km}$ oflinear transects were analyzed at the municipalities of Baudo and Certegui. We documented 42 records of presence of the species, resulting in a relative abundance of 3.0 individuals $/ \mathrm{km}$ with confidence intervals of 2.7 to 4.0 individuals $/ \mathrm{km}$. The study area was characterized by a high heterogeneity including contrasting vegetation types. We report 42 tree species associated with the transects; $48 \%$ of them represented genera previously reported in the diet of T. pecari. Palms were particularly important in the analyzed plots adding $20 \%$ of the arboreal elements, with Socratea sp. accounting for $8.57 \%$ of the tree elements. Industrial mining and expansion of the agricultural boundary of nontraditional plants were identified as major threats for the species at the study sites.

Keywords: Abundance; Choco; Conservation; Ecological characterization; Transects; Transects; With-lipped peccary.
1. Grupo de Investigación en Manejo de Fauna Silvestre del Chocó, Universidad Tecnológica del Chocó, Quibdó, Colombia.e-mail:1epamo8@gmail.com

2. Investigadora, Instituto de Investigaciones Ambientales del Pacífico (IIAP), Quibdó, Colombia.

e-mail: caparra@yahoo.es

3. Department of Biological Sciences, Texas Tech University.

e-mail: hugo.mantilla@ttu.edu Grupo de Investigación en Conocimiento, manejo y conservación de los ecosistemas delChocó Biogeográfico.

Recibido: 10 de febrero de 2010

Aceptado: 25 de febrero de 2010 


\section{INTRODUCCIÓN}

El Tatabro sin collar (Tayassu pecari) (Artyodactila: Tayassuidae) se distribuye en la región neotropical, en una gran variedad de ecosistemas por debajo de los $1,800 \mathrm{~m}$, desde el sureste de México en los estados de Oaxaca y Veracruz a lo largo de toda Centro y Sur América hasta el Norte de Argentina. Adicionalmente, la especie ha sido también introducida en Cuba (Wilson y Reeder 2005). En Colombia $T$. pecari habita todo el país y ha sido reportada en el Chocó para el municipio de Riosucio: P.N.N. Katíos, Corregimiento El Gilgal, en la vereda Marsella parte alta del río Cutí (MuñozSaba y Alberico, 2004).

Aunque Rodríguez-Mahecha et al. (2006) no reportan a $T$. pecari entre los mamíferos amenazados de Colombia, la especie se encuentra en la categoría de amenaza de Menor Riesgo (LR nt) según la Unión Internacional para la Conservación de la Naturaleza(UICN) (2003) y ha sido incluida en la lista de la Convención sobre el Comercio Internacional de Especies Amenazadas de Fauna y Flora Silvestres (CITES) en su Apéndice II (Morales-Jiménez et al. 2004).

Considerado como un animal de gran importancia dentro de la actividad de caza, $T$. pecari es y ha sido un recurso cultural y alimentario muy importante en el desarrollo de las comunidades negras e indígenas en la región (Sowls 1997). A pesar de que es considerado un elemento común de la fauna local, en los últimos años los cazadores del Chocó Central han informado una disminución en sus poblaciones representada en una mayor dificultad para su captura (López et al. 2006). Esta situación se ha visto agravada por la escasez de frutos consecuencia aparente de la tala selectiva de maderas, que fuerzan a las manadas a efectuar mayores desplazamientos en procura de recursos alimenticios, exponiéndolas de una mayor manera a la caza como ha sido verificado en otras latitudes (Altrichter y Almeida 2002). Sumado a esto, la extracción de metales preciosos producto de la actividad minera con retroexcavadora ha causado la degradación rápida e irreversible de sus hábitats; estos factores han sido sugeridos como causantes de la extinción de poblaciones de $T$. pecari a nivel local y regional (Ruiz y García 2004).

Para la región del Chocó Central, que concentra la mayor parte de los asentamientos humanos del Chocó Biogeográfico colombiano no existe a la fecha una estimación de las poblaciones de tatabros sin collar T. pecari que esté sustentada en una metodología repetible, permitiendo así la comparación geográfica y temporal de las abundancias relativas de esta especie que permita establecer un adecuado plan de manejo. El propósito de este estudio fue estimar la abundancia relativa y caracterizar el hábitat del tatabro sin collar en cuatro localidades de dos municipios de la zona centro del Chocó utilizando transectos lineales y parcelas de vegetación.

Area de estudio. El estudio se realizó en los municipios del
Medio Baudó y Cértegui, ubicados en la zona centro del Chocó, Colombia. El municipio del Medio Baudó, está localizado en la parte central del departamento del Chocó. Su cabecera municipal es Puerto Meluk, población emplazada en la margen izquierda del río Baudó a los $05^{\circ} 11^{\prime}$ '66.5' ' de latitud norte y $76^{\circ} 57^{\prime} 28.7^{\prime}$ ' de latitud oeste, a una distancia de $95 \mathrm{~km}$ de Quibdó aproximadamente. Este ente territorial tiene una extensión de 1.390,6 km². Está integrado por un sistema de pequeños asentamientos localizados sobre la cuenca del río Baudó y sus tributarios; la mayor parte del territorio está cubierto de bosques y selva (EOT 2006).

El municipio de Cértegui, se encuentra localizado en la subregión del San Juan. Tiene una extensión de $342 \mathrm{~km}^{2}$. La cabecera del municipio es Cértegui, la que se encuentra ubicada a los 5 41' 41' 'Ny los 76 39' 40', W, en la confluencia de los ríos Quito y Cértegui, (EOT 2006). Ambos municipios corresponden a tierras bajas donde predominan las selvas hiperhúmedas con temperatura media anual alrededor de $26.7^{\circ} \mathrm{C}$, máxima de $34.9^{\circ} \mathrm{C}$ y mínima de $22^{\circ} \mathrm{C}$ (Rangel 2004 ), caracterizadas por una altísima precipitación media anual que en Cértegui llega a los $7.000 \mathrm{~mm}$.

\section{MATERIALES Y MÉTODOS}

Con el fin de estimar la abundancia relativa y caracterizar el hábitat del tatabro sin collar T. pecari en la región del Chocó Central, se llevaron a cabo ocho faenas de campo en las localidades de Puerto Meluk y Curundó La Banca, ubicados en el municipio del Medio Baudó y en los corregimientos de La Variante y La Toma en el municipio de Cértegui, entre los meses de octubre y noviembre de 2009. La estimación de la abundancia se basó en muestreos de transectos lineales, de longitudes que variaron entre 1.4 y $2.8 \mathrm{~km}$. Las diferencias en longitud estuvieron dictadas por la variable fisiográfía del terreno en los puntos de muestreo. Para la ubicación de cada transecto se tuvieron en cuenta los siguientes criterios siguiendo lo propuesto por Ojasti (2000): 1) lugares en que los pobladores de cada una de las comunidades reportaron la presencia de los pecaríes, 2) hábitats con condiciones adecuadas para la especie, según lo reportado en la literatura, 3) condición del terreno y accesibilidad y 4) avistamientos previos por parte de los investigadores (individuos, huellas, heces, frutos, comederos).

Registro de datos de presencia. Fueron considerados como datos de presencia de la especie: 1) huellas impresas de manera clara sobre el sustrato del transecto, a las cuales se les tomaron impresiones en molde con yeso odontológico de acuerdo con la metodología reportada por Aranda (2000). Los moldes fueron evaluados en forma y tamaño mediante la utilización de la guía de campo de huellas propuesta por Morales-Jiménez et al. (2004); 2) frutos parcialmente consumidos en los cuales se identifico con claridad la mordida típica 


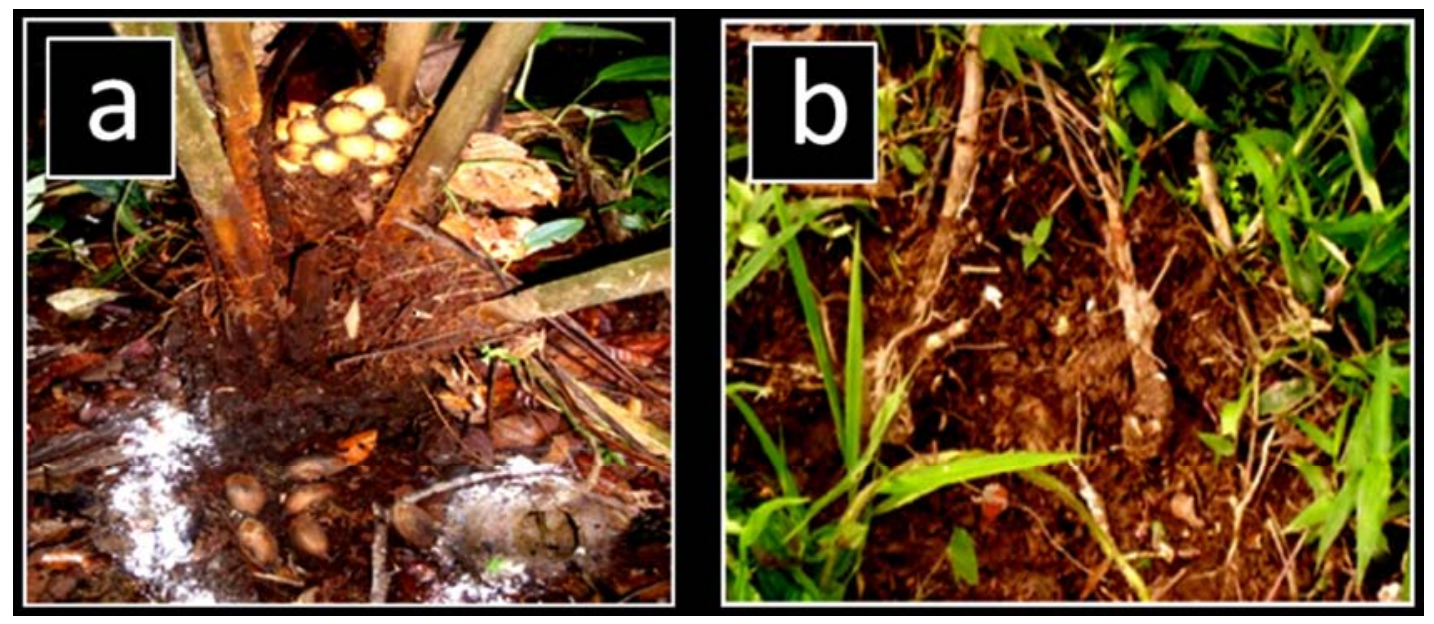

Figura 1. A. Comedero del tatabro sin collar en palma de táparo Orbignya cuatrecasana registrado en área de cultivo en el corregimiento de Curundó La Banca, municipio de Medio Baudó. B. Comedero del tatabro sin collar en yuca, Manihot sculenta, registrado en área de cultivo en el corregimiento de La Variante, municipio de Cértegui.

de los tatabros, 3) comederos, definidos como sitios de acumulación de recursos explorados por los animales (Figuras 1 A y B). Finalmente, fueron tenidas en cuenta como registros las heces depositadas sobre los transectos.

Se definió como presencia de un único individuo: 1) huellas individuales, 2) senderos de huellas independientes, 3) frutos hallados individualmente, 4) comederos, 5) heces. Cada una de estas evidencias de presencia fue georeferenciada sobre el transecto con la ayuda de un GPS Garmin Colorado 400. Cada transecto se recorrió una sola vez para evitar sobrestimación de los registros (CODECHOCO-UTCH 2009).

Estimación de la abundancia. La abundancia relativa de T. pecari se estimó de acuerdo a la metodología de transecto definida por Ojasti (2000), la cual consiste básicamente en el registro del número de evidencias de presencia de la especie en rutas o transectos, dividida por la longitud de los mismos y expresada en número de individuos $/ \mathrm{km}$ recorrido, con sus respectivas medidas de desviación (varianza, desviación estándar) e intervalos de confianza al 95\%. La abundancia relativa fue calculada individualmente para cada municipio y luego se calculó la abundancia relativa promedio para toda la zona de estudio (involucrando las abundancias de los dos municipios). La media y la varianza se calcularon con base en las medidas de tendencia central y de desviación estándar con sus respectivos límites de confianza al 95\%, con la ayuda de SPSS versión 15.0 disponible en http://www.spss.com. version 15

Caracterización de hábitat. Para la caracterización del hábitat de T. pecari en la zona de estudio, se describió la vegetación asociada con cada punto del transecto en el cual se registró evidencia de presencia de la especie, basados en la metodología propuesta por Gentry (1982) con algunas modificaciones en cuanto al área de las parcelas. Para cada línea de transecto donde efectivamente se observó indicios de la especies se demarcó una parcela de $2 \mathrm{~m}$ de ancho por $50 \mathrm{~m}$ de largo, en donde se registraron e identificaron todas las especies arbóreas con Diámetro a la Altura del Pecho (DAP) $\geq 10 \mathrm{~cm}$. Para cada individuo se registraron su altura total y diámetro de la copa; de igual forma se tuvieron en cuenta algunas variables físicas como altitud, pendientes y distancias entre el transecto y cuerpo de agua más cercano, datos que formarán parte de análisis futuros y no son incluidos en este trabajo (Figura 2).

La caracterización vegetal incluyó: la determinación taxónómica de especies vegetales presentes en los sitios donde se confirmó la presencia de $T$. pecari, además se tomaron registros fotográficos de la zona, así como la descripción de las posibles especies vegetales utilizadas como fuente de alimentación y refugio por la especie basados en encuestas directas a cazadores en el área de estudio.

\section{RESULTADOSYDISCUSIÓN}

Se registró un total de 42 indicios de presencia del tatabro sin collar, para un total de $16.4 \mathrm{~km}$ de transecto en línea analizados. Las huellas constituyeron el indicio predominante para todas las localidades analizadas sumando $83.3 \%$ de registros seguidas de $9.5 \%$ de los comederos, $4.8 \%$ los frutos y las heces $2.4 \%$. Reportamos una abundancia relativa promedio para la zona de 3 individuos $/ \mathrm{km}$, con una desviación estándar de 0.07 e intervalos de confianza al $95 \%$ que van desde 2.7 hasta 4 individuos $/ \mathrm{km}$. El mayor número de registros se dio en las localidades de la Variante, municipio de Cértegui y Curundó la Banca del Medio Baudó con 9.29 y 5.5 indivi- 
Abundancia relativa y caracterización del hábitat de Tayassu pecari. L Palacios-Mosquera, et al.

Tabla 1

Abundancia relativa y número de rastros del tatabro sin collar en las localidades Medio Baudó y Cértegui

\begin{tabular}{llcccc}
\hline Municipio & Localidad & No huellas & $\begin{array}{c}\text { Distancia/recorrido } \\
\text { km }\end{array}$ & $\begin{array}{c}\text { Abundancia relativa } \\
\text { Abundancia } \\
\text { relativa promedio }\end{array}$ \\
\hline \multirow{2}{*}{ Medio Baudó } & Puerto & 2 & 2.0 & 1,00 & \\
& Meluk & 0 & 2.0 & 0,00 & 2,50 \\
& Curundo & 8 & 2.3 & 3,48 & \\
Cértegui & La Banca & 11 & 2.0 & 5,50 & \\
& Variante & 13 & 1.4 & 9,29 & 3,40 \\
& & 1.7 & 4,10 & \\
Promedio & La Toma & 1 & 2.8 & 0,36 & 3,00 \\
Varianza & 0 & 2.2 & & 0,01 \\
Desviación estándar & 42 & 16.4 & & 0,07 \\
Límite superior (95\%) & & & & 4,00 \\
Límite inferior (95\%) & & & & 2,71 \\
\hline
\end{tabular}

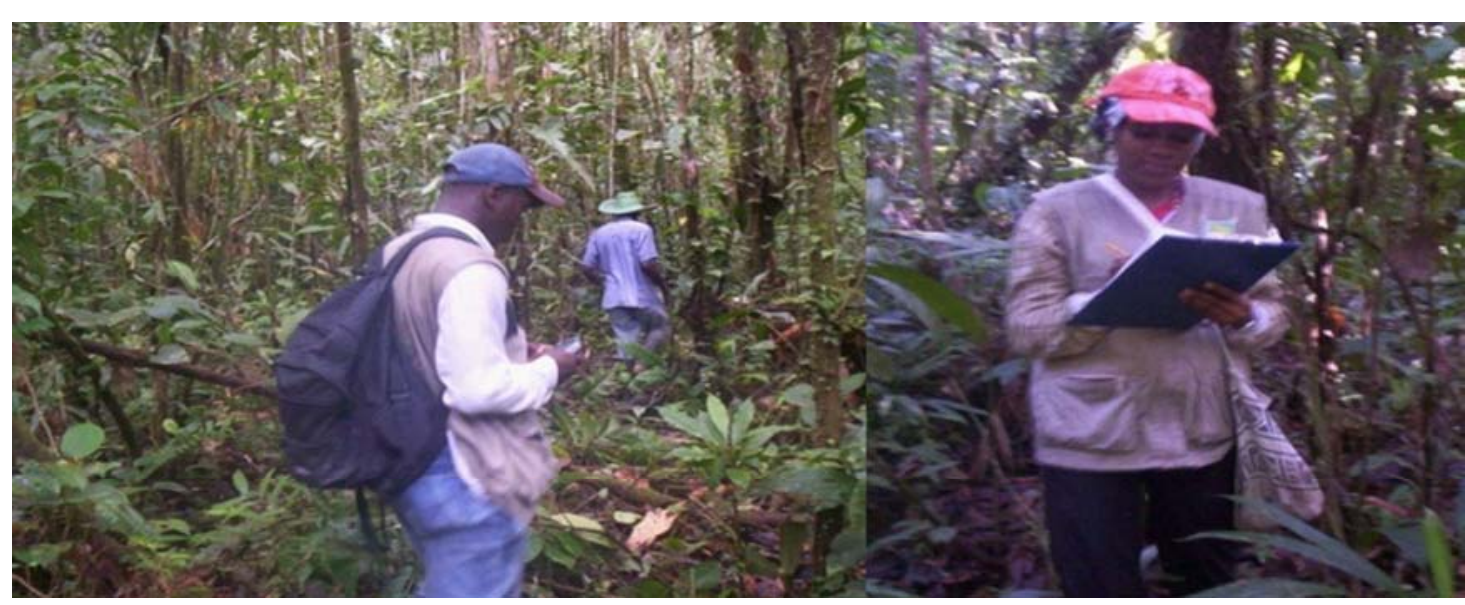

Figura 2. Panorámica de los bosques de la zona donde habita el tatabro sin collar, registro de huellas y características de la vegetación.

duos $/ \mathrm{km}$, respectivamente. De igual forma la mayor abundancia relativa promedio fue para Cértegui con 3.4 individuos $/ \mathrm{km}$, mientras que para Medio Baudó el registro fue más bajo con 2,5 individuos $/ \mathrm{km}$ ) (Tabla 1 ).

Se observaron 42 indicios del tatabro sin collar y se estableció que el valor más alto en relación con la abundancia relativa por municipios para toda la zona, los presentaron las localidades de la Variante, municipio de Cértegui y Curundó la Banca del Medio Baudó con 9.29 y 5.5 individuos $/ \mathrm{km}$, en donde los avistamientos de huellas constituyeron el indicio predominante; de igual forma, la mayor abundancia relativa promedio fue para Cértegui con 3.4 individuos $/ \mathrm{km}$, mientras que para Medio Baudó el registro fue más bajo (2.5 individuos $/ \mathrm{km}$ ) (Tabla 1$)$.

Características del hábitat para Tayassu pecari. Los dos municipios analizados constituyen un mosaico de coberturas vegetales asociadas con diferentes grados disturbio así: 1) Bosque primario estratificado, 2) Bosques intervenidos, que incluyen bosques en regeneración, 3) Rastrojo y 4) Áreas cultivadas. Se identificaron un total de 42 especies arbóreas (DAP $>10 \mathrm{~cm}$ ) incluidas en las parcelas de muestreo (Tabla 2). En los bosques primarios predominaron como elementos arbóreos: Brosimun utile, Castilla elastica, 
Bioetnia Volumen 7 № 1 (enero-junio), 2010

Tabla 2

Clasificación taxonómica, número y porcentaje de elementos arbóreos más importantes (DAP $>\mathbf{1 0} \mathrm{cm}$ ) incluidos en las parcelas de vegetación asociadas con los transectos

\begin{tabular}{|c|c|c|c|c|c|c|c|}
\hline \multicolumn{2}{|c|}{ Nombre } & \multirow{2}{*}{$\begin{array}{c}\mathrm{N}^{\circ} \\
\text { individuos }\end{array}$} & \multirow[t]{2}{*}{$\%$} & \multicolumn{2}{|c|}{ Nombre } & \multirow{2}{*}{$\begin{array}{c}\mathbf{N}^{\circ} \\
\text { individuos }\end{array}$} & \multirow[t]{2}{*}{$\%$} \\
\hline común & científico & & & común & científico & & \\
\hline Palma zancona & Socrateasp * & 9 & 8.57 & Guascablanco & Guethalsiasp & 2 & 1.90 \\
\hline Pacó & Gustaviasuperba* & 6 & 5.71 & Hormigónblanco & Carapaguianensis * & 2 & 1.90 \\
\hline Yarumo & Pouroumachocoana & 6 & 5.71 & Manchará & Vismialatisepala & 2 & 1.90 \\
\hline Nuanamo & Iryantherasp & 5 & 4.76 & Sajo & Campnospermapanamensis & 2 & 1.90 \\
\hline Palma meme & Wettiniaquinaria & 5 & 4.76 & Aliso & Alnusglutinosa & 1 & 0.95 \\
\hline Palma mil peso & Oenocarpusbataua * & 5 & 4.76 & Boteco & Matisia sp. & 1 & 0.95 \\
\hline Algodoncillo & Asclepiascurassavica & 4 & 3.81 & Carra & Huberodendronpatinoi & 1 & 0.95 \\
\hline Caimito & Pouteria $s p .^{*}$ & 4 & 3.81 & Castaño & Compsoneuraotopa & 1 & 0.95 \\
\hline Guasca & Eschwilerapittieri & 4 & 3.81 & Castañoblanco & Matisiacastanno & 1 & 0.95 \\
\hline Otobo & Otobalatialata * & 4 & 3.81 & Cauchillo & Castillaelastica & 1 & 0.95 \\
\hline CaimitoTáparo & Micropholissp & 3 & 2.86 & Cedro macho & Tapiriraguianensis & 1 & 0.95 \\
\hline Chanó & Sacoglottisprocera & 3 & 2.86 & Costillo & SagotiaracosaBaill, & 1 & 0.95 \\
\hline Guamocajeta & Inga coruscansWilld * & 3 & 2.86 & Guayacan & Minquartiaguianensis & 1 & 0.95 \\
\hline Laurel & Cordiaalliodora * & 3 & 2.86 & Guayavo & Eugenia $s p *$ & 1 & 0.95 \\
\hline Lechero & Brosimun utile * & 3 & 2.86 & Hueso & Lindackeriasp & 1 & 0.95 \\
\hline Carbonero & Licaniadurifolia * & 2 & 2.86 & Moro & Miconiasp & 1 & 0.95 \\
\hline Dormilon & Pentaclethramacroloba & 3 & 2.86 & Otobo & Otobalatialata * & 1 & 0.95 \\
\hline Algarrobo & Hymenaeaoblongifilia * & 2 & 1.90 & Palo Perico & Indeterminada & 1 & 0.95 \\
\hline Anime & Dacryodesoccidentalis & 2 & 1.90 & Palma chacarrá & Bactrisborronoi * & 1 & 0.95 \\
\hline Guamo & Inga cocleensis & 2 & 1.90 & Palma cabeci- & Minicariasaccifera & 1 & 0.95 \\
\hline Guamorosario & Inga acrecephala & 2 & 1.90 & negro & & & \\
\hline
\end{tabular}

* Provienen de géneros cuyas semillas y frutos son consumidos por pecaríes en otras localidades del neotrópico según Beck (2005)

Guethalsia sp., Huberodendron patinoi, Hymenaea oblongifilia, Iryanthera sp., Licania durifolia, Lindackeria sp., Otoba latialata, Pouteria caimito, Sagotia racosa; mientras que en los bosques intervenidos se destacaron: Asclepias curassavica, Carapa guianensis, Gustavia superba, Pourouma chocoana y Vismia latisepala; finalmente las áreas de rastrojo incluyeron como elementos arbóreos Alnus glutinosa, Guarea manchare, Inga cocleensis, Matisia sp., Miconia sp., Pentaclethra macroloba y $P$. chocoana. De 42 especies registradas, 15 sumaron $63 \%$ de los elementos arborescentes identificados, destacándose la Palma Zancona, Socratea sp. que por sí sola sumó $8.6 \%$ de los registros, seguida del Pacó, G. superba y el Yarumo $P$. chocoana ambas aportando 5.7\% a los elementos arbóreos. Por su parte las áreas cultivadas incluyeron plantaciones de pan coger: yuca, Manihot sculenta; plátano, Musa sapientum; banano, M. paradisiaca; ñame, Dioscorea alata y maíz, Zea mays y asociados con estos cultivos frutales como el cacao, Theobroma cacao y chontaduro Bactris borronoi y $B$. gasipaes.

Disponibilidad de alimento. Las palmas han sido men- cionadas como especies clave para los pecaríes (Terborgh 1992). Según Bodmer et al. (1997) y al parecer palmas y pecaríes están entrelazados en una relación coevolutiva que implica la producción de semillas muy duras por parte de las palmas y mecanismos de ingestión/digestión especializados por parte de los pecaríes. En nuestros muestreos se registró la presencia de seis especies de palmas: B. borronoi, $B$. gasipaes, Minicaria saccifera, Oenocarpus bataua, Socratea sp. y Wettinia quinaria sumaron $20 \%$ de los elementos arbóreos (DAP $>10 \mathrm{~cm})$, siendo Socratea sp. el elemento aborescente más común en las parcelas asociadas con los transectos (8.6\%), como se mencionó con antelación. Algunos autores sugieren que la utilización de semillas duras de palmas por parte $T$. pecari es un recurso adaptativo para evitar competencia con otros ungulados frugívoros como dantas (Tapirus bairdii) y saínos (T. tajacu) (Kiltie 1982, Bodmer 1989). Dentro de los elementos arbóreos destacados por su número (5.7\%) se reporta también la Lecithidaceae $G$. superba cuya dispersión es facilitada por $T$. pecari en otras regiones del neotrópico (Beck 2005).

Aunque no se presenta como un dato conclusivo, es 
Abundancia relativa y caracterización del hábitat de Tayassu pecari. L Palacios-Mosquera, et al.

Tabla 3

Frutos consumidos por el tatabro sin collar reportados por habitantes de la región

\begin{tabular}{llll}
\hline Nombre vulgar & Nombre científico & Familia & Parte de la planta que consumen \\
\hline Árboles & & & \\
Otobo & Otoba latialata & Myristicaceae & Semillas \\
Coronillo & Bellucia axinanthera & Melastomataceae & Frutos \\
Caimito & Pauteria caimito & Sapotaceae & Semilla \\
Cultivos & Manihot sculenta & Euphorbiaceae & Frutos \\
Yuca & Musa sapientum & Musaceae & Frutos \\
Plátano & Musa paradisiaca & Musaceae & Frutos \\
Banano & Bactris gasipaes & & \\
Palmas & Oenocarpus bataua & Arecaceae & \\
Palma de chontaduro & Arecaceae & Semilla \\
Palma mil peso & Orbignya cuatrecasana & Arecaceae & Frutos \\
Palma táparo & Astrocaryum standleyanum & Arecaceae & Frutos \\
Palma wuerregué & Bactris borronoi & Arecaceae & Semilla \\
Palma chascarrá & & & \\
\hline
\end{tabular}

interesante anotar que cuando contrastamos la lista de especies arbóreas identificadas en las parcelas asociadas con nuestros transectos, con aquella de especies que son consumidas por los pecaríes según datos de Beck(2005), encontramos que 16 de las 42 especies identificadas corresponden a géneros usados por los tatabros en su dieta en otras localidades del neotrópico (especies señaladas por un asterisco $\left({ }^{*}\right)$ en la Tabla 2); estas 16 especies suman $48 \%$ de los elementos arbóreos registrados lo que indica cierto grado de selección de hábitat por parte de los tatabros sin collar para el área de estudio. De manera adicional y de acuerdo con la evidencia colectada y encuestas con los cazadores locales se determinó el consumo de 12 items vegetales en la dieta de T. pecari para la zona de estudio que son listados en la Tabla 3.

El uso de especies cultivadas como M. suculenta por parte de T. pecari plantea un conflicto de conservación para la especie en el área de estudio, donde los habitantes manifestaron que el tatabro sin collar destruye los cultivos de esta planta al igual que plantas silvestres de táparo, $O$. cuatrecasana que son aprovechadas por los habitantes de la zona. Plantas de $O$. cuatrecasana constituyeron los dos de los comederos de T. pecari registrados en este estudio.

Presión del hábitat del Tayassu pecari. Aunque en la zona de estudio aún existen relictos de vegetación natural en buen estado de conservación, una buena parte de sus bosques son secundarios con diferentes estados de regeneración y/o han sido altamente modificados por las actividades humanas como la agricultura, tala y la minería. Consideramos que se deben tomar medidas de manera urgente tendientes a mitigar el impacto generado por estas actividades, en especial la extracción de metales precioso como oro y platino, y la extracción indiscriminada de productos maderables que aunque constituyen la principal fuente de ingreso económico en la región, ha producido la degradación y/o alteración irreversible de muchos ecosistemas sobre todo en los municipio de Unión Panamericana y Cértegui.

\section{CONCLUSIONES}

La información aquí reportada constituye un punto de partida indispensable en el seguimiento de la dinámica poblacional de esta especie importante por su función natural en los ecosistemas como predador y dispersor de semillas y elemento fundamental de la economía rural de las poblaciones chocoanas para una de las zonas del departamento con mayor número de asentamientos humanos. Al ser comparados con estudios realizados en otras localidades del neotrópico, los datos de densidad poblacional arrojados por nuestro estudio caen dentro de los rangos indicados para localidades donde la especie se encuentra bajo alta presión de cacería y por debajo de densidades relativas reportadas para localidades de la Amazonía de bosques en buen estado de conservación y menor presión antrópica (Gottdenker y Bodmer 1998). De lo anterior se colige que las poblaciones de esta especie están siendo altamente afectadas por la acción de actividades antrópica típicas en la región. La minería, la tala indiscriminada, la expansión de la frontera agrícola de cultivos no tradicionales y la ganadera se constituyen en las principales 


\section{Bioetnia Volumen 7 № 1 (enero-junio), 2010}

causas que ponen en riesgo las poblaciones naturales de $T$. pecari en la zona de estudio.

Se recomiendan explorar con más detalle el estado poblacional de esta especie involucrando variables como radios de sexo, ciclos reproductivos, dieta, fenología, al igual que variables abióticas como variaciones de temperatura, precipitación y fase lunar sobre la movilidad de los grupos de tatabros que ayuden a entender mejor su demografía en la zona de estudio. También consideramos importante involucrar a la comunidad local, en especial a los niños y jóvenes quienes en conjunto con los cazadores y con el acompañamiento de la autoridad ambiental, deben ser parte fundamental del proceso de manejo de la especie.

dencia de la presencia de las especies objeto de esta investigación en la zona de estudio. Con base en lo anterior se colige que las poblaciones de estas especies están muy afectadas por la acción de actividades antrópicas típicas de la región.

La minería, el ruido producido por las motosierras al extraer la madera, la expansión de la frontera agrícola, ganadera y de cultivos no tradicionales en la zona de estudio, constituyen las principales causas que han llevado a poner en riesgo las poblaciones naturales de los puercos de monte, porque el número de individuos del tatabro sin collar (T. pecari) por kilometro fue relativamente bajo.

El hallazgo de indicios de la presencia de la especie ( $T$. pecari) en lugares donde se encontraron relictos o fragmentos de vegetación en buen estado de conservación hasta áreas de cultivos, deja manifiesto que el tatabro sin collar explota diversos tipos de hábitat dentro de los ecosistemas de las zonas de estudio.

Se recomienda explorar con más detalle el estado poblacional de estas especies, involucrando variables como las características reproductivas, dieta alimenticia de los puercos de monte y las diferentes épocas climáticas de mayor actividad. Además se propone desarrollar más muestreos y clarificar la influencia de la actividad lunar sobre la movilidad de estas especies, con el fin de disminuir el sesgo sobre la estimación de las poblaciones naturales. También es importante que los niños y jóvenes conozcan las experiencias de los cazadores sobre los puercos de monte, con el acompañamiento de la autoridad ambiental, que involucre el proceso de manejo de fauna silvestre, pues son ellos los futuros usuarios de los recursos naturales y quienes requieren con mayor precisión estos conocimientos.

\section{AGRADECIMIENTOS}

Los autores agradecemos a la corporación CODECHOCO, al IIAP, por el apoyo otorgado para llevar a feliz término el estudio, a Paola Cano por su ayuda en logística, a los consejos comunitarios y habitantes de los dos municipios, por su colaboración en campo y compartir sus conocimientos y experiencias sobre la fauna objeto de estudio. Este proyecto es parte del Plan de Manejo para la Conservación de los cerdos de monte (Tayassu tajacu y $T$. pecari) y los osos perezosos (Bradypus variegatus y Choloepus hoffmanni) en seis municipios del departamento del Chocó, Colombia.

\section{LITERATURA CITADA}

Aranda, M. 2000. Huella y rastros de los mamíferos medianos y grandes de México. Veracruz: Instituto de Ecología AC; 212 p.

Altrichter, M. 2000. Importancia de los mamíferos silvestres en la dieta de pobladores de la península de Osa. Rev Mex Mastozool 4: 99-107.

Bodmer, R. E., K. L. Sowls. 1996. El pecarí de collar. En: W. Oliver (ed.). Plan de acción y evaluación de la condición actual de los pecaries. Gland: IUCN; p. 5-15.

Bodmer, R. E. 1989. Frugivory in amazon ungulates. Thesis. Cambridge: University of Cambridge. 311 p.

Bodmer, R. E, R. Aquino, P. E. Puertas, C. J. Reyes, T. G. Fang, N.L. Gottdenker. 1997. Manejo y uso sustentable de pecaries en la Amazonía peruana. Lima: Paper ocasional $\mathrm{N}^{\circ} 18$ de la Comisión de Supervivencia de Especies; 102 p.

CODECHOCO-UTCH. 2009. Plan de manejo y conservación de la guagua (Cuniculus paca) y el chigüiro (Hydrochoerus isthmius) en los ecosistemas del Medio y Bajo Atrato, Chocó, Colombia. Quibdó: CODECHOCOUTCH; $156 \mathrm{p}$.

Fragoso, J.M.V. 1998. White-lipped peccaries and palms on the Ilho de Maracá, In: W. Milliken, J. Ratter(eds.). Maracá: The biodiversity and environment of an Amazonian rain forest. New York: John Witey; p. 151-63.

Gentry, A.H. 1982. Pattems of neotropical plant species. Evol Biol 15: 1-84.

Kiltie, R. A. 1982. Bite forces as basis for niche differentiation between rain forest peccaries (Tayassu tajacu and T. pecari). Biotropica 14: 188-95.

López, M.T., M. Altrichter, J. Sáenz, E. Eduarte. 2006. Valor nutricional de los alimentos de Tayassu pecari (Artiodactyla: Tayassuidae) en el Parque Nacional Corcovado, Costa Rica. Rev Biol Trop. 54: 687-700.

Muños-Saba, M. Alberico. 2004. Mamíferos en el Chocó Biogeográfico. En: Rangel-Ch., O. J. (Eds.). Diversidad Biótica IV. El Chocó Biogeográfico/ Costa Pacifica. Bogotá: Universidad Nacional de Colombia; 559 pp.

Noss A.J., Rosa L. Cuéllar. 2008. La sostenibilidad de la cacería de Tapirus terrestris y de Tayassu pecari en la tierra comunitaria de origen isoso: el modelo de cosecha unificado. Mastozool Neotrop. 15: 241-52.

Rodríguez-M., J. V., M. Alberico, F. Trujillo, J. Jorgenson (Eds.). 2006. Libro Rojo de Mamíferos de Colombia. Serie Libro Rojo de Especies Amenazadas de Colombia. Bogotá, DC: Conservación Internacional Colombia y Ministerio de Medio Ambiente Vivienda y Desarrollo Territorial; 433 pp.

Ojasti, J. 2000. Manejo de fauna silvestre neotropical. En: F. Dallmeier (ed.). SI/MAB Series $N^{\circ}$ 5. Washington, DC: Smithsonian Institution/MAB Biodiversity Program; 309 pp.

Rangel, O. J. 2004. Colombia diversidad biótica IV: El Chocó Biogeográfico/ Costa Pacífica. Bogotá, DC: Universidad Nacional de Colombia.

Ruiz, F.R., M.V. García. 2004. Abundancia poblacional, movimientos y uso de hábitat del tapir (Tapirus bairdii) en el Parque Nacional Laguna Lachua, Alta Verapaz, Guatemala. Informe Final. Managua: Universidad de San Carlos de Guatemala; 51 p.

Sowls, L.K. 1997. Javelinas and other peccaries, their biology, management and use. 2nd ed. Tucson: The University of Arizona; $324 \mathrm{pp}$.

Terborgh, J. 1992. Diversity and the tropical rain forest. New York: Scientific American Library; 242 pp.

Wilson, D, M. Reeder (Eds.). 2005. Mammals species of the world: A taxonomic and geographic reference. 3rd ed. Baltimore: The Johns University Press; $2142 \mathrm{pp}$.

Zapata-Ríos, G. Edison-Araguillin, Y. Jeffrey, P. Jorgenson. 2006. Caracterización de la comunidad de mamíferos no voladores en las estribaciones orientales de la cordillera del Kutukú, Amazonía ecuatoriana. Mastozool Neotrop. 13: 227-38. 